Rev. salud pública. 13 (2): 219-228, 2011

\title{
Aptidão física relacionada a saúde em adolescentes brasileiros residentes em áreas de médio/baixo índice de desenvolvimento humano
}

\section{Health-related physical fitness in Brazilian adolescents from areas having a medium/low Human Development Index}

\author{
Edio Luiz Petroski ${ }^{1}$, Adelson Fernandes da Silva ${ }^{2}$, Adriana Bispo Rodrigues ${ }^{2}$ \\ e Andreia Pelegrini ${ }^{3}$
}

\begin{abstract}
1 Universidade Federal de Santa Catarina. Programa de Pós-Graduação em Educação Física Florianópolis, SC. Brasil. petroski@cds.ufsc.br

2 Universidade Estadual de Montes Claros. Januária, MG. Brasil. adelson_fs@yahoo.com.br, Adriana_br@yahoo.com.br

3 Universidade Estadual do Oeste do Paraná. Centro de Ciências Humanas. Marechal Cândido Rondon, PR. Brasil. a.pelegrini@yahoo.com.br

Recebido em 22 Novembro 2010/Enviado para Modificaçao em 1 Abril 2011/Aprovado em 15 Abril 2011
\end{abstract}

\section{RESUMO}

Objetivo Foi avaliar a aptidão física relacionada à saúde (AFRS) em adolescentes em adolescentes de uma cidade com médio/baixo índice de desenvolvimento humano. Métodos Participaram do estudo 627 adolescentes (266 rapazes e 361 moças), de 14 a 17 anos. Foram mensuradas: massa corporal, estatura, dobras cutâneas e o perímetro da cintura. A bateria de aptidão física e os pontos de corte para critérios de saúde sugeridos pelo Physical Best (1988), foram utilizados.

Resultados Não atingiram os critérios mínimos à saúde: composição corporal $25 \%$; flexibilidade 40,8 \%; força/resistência muscular 98,5 \%, e, aptidão cardiorrespiratória $35,4 \%$. Na classificação geral, nos três testes motores, foi observada alta proporção de adolescentes (masculino 99,6 \% e feminino $100 \%$ ), que não atenderam os critérios pré-estabelecidos para um nível satisfatório de aptidão física.

Conclusão Independentemente do sexo e idade, os adolescentes apresentaram baixa AFRS. Neste sentido, acredita-se que seja de fundamental importância à implementação de intervenções direcionadas à população em idade escolar.

Palavras-chave: Aptidão Física, atividade motora, estudantes e condições sociais (fonte: DeCS, BIREME).

\section{ABSTRACT}

Objective Evaluating health-related, physical fitness (HRPF) in adolescents from a town having a medium/low Human Development Index (HDI). 
Methods This was a descriptive study involving 627 adolescents (266 boys and 361 girls) from public schools aged 14 to 17. Anthropometric measurements were obtained and the students were given motor tests analysed according to the cut-off points suggested by US Physical Best fitness standards (1988).

Results Low physical fitness was observed amongst the students being studied, thereby presenting a health risk in terms of flexibility $(40.8 \%)$, muscle strength/endurance (98.5\%) and cardio respiratory fitness (35.4\%); $25 \%$ of the students did not fulfil the body composition criteria. Overall classification in the three motor tests revealed a high percentage of students from both genders (boys: $99.6 \%$ and girls: $100 \%$ ) who did not fulfil the preestablished criteria for having a satisfactory level of physical fitness.

Conclusion The results indicated that implementing intervention aimed at the school-age population having poor HRPF is of fundamental importance.

Key Words: Motor activity, physical fitness, student, social conditions (source: $M e S H, N L M$ )

\section{RESUMEN}

Capacidades físicas relacionadas con la salud de adolescentes brasileños residentes en áreas con índice de desarrollo humano medio/bajo

Objetivo Evaluar las capacidades físicas relacionadas con la salud (AFRS) en adolescentes de una ciudad con índice de desarrollo humano medio/bajo.

Método Participaron en el estudio 627 adolescentes (266 de sexo masculino y 361 de sexo femenino) entre 14 y 17 años. Se realizaron las siguientes medidas: masa corporal, pliegues cutáneos y perímetros de cintura. Se utilizo la batería de capacidades físicas y los puntos de corte sugeridos por el Physical Best (1998).

Resultados No se obtuvieron los criterios mínimos de salud: composición corporal $25 \%$; flexibilidad 40,8 \%; fuerza/resistencia muscular 98,5\%, y; capacidad cardiorespiratoria 35,4\%. En general, en las tres pruebas motoras, se observó un alto número de adolescentes (96,6\% masculinos y $100 \%$ femeninos) que no consiguieron los criterios preestablecidos para un nivel satisfactorio de capacidades físicas.

Conclusión Independientemente del sexo y edad, los adolescentes presentaron un bajo AFRS. En este sentido se piensa que es de fundamental importancia añadir intervenciones direccionadas a la población escolar.

Palabras Clave: Acondicionamiento físico, estudiantes, actividad motora y condiciones sociales (fuente: DECS, BIREME).

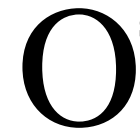

s avanços científicos e tecnológicos na sociedade contemporânea proporcionaram melhoras significativas e indiscutíveis na qualidade de vida das pessoas. Por outro lado, ocasionaram redução significativa no envolvimento com atividade física e esporte, fragilizando os componentes da aptidão física relacionada à saúde (AFRS) (1).

Os componentes da AFRS devem contemplar indicadores da capacidade cardiorrespiratória, força/resistência muscular, flexibilidade e gordura corporal (2). Estes, por sua vez, estão relacionados ao estado de saúde, seja nos aspectos da prevenção e redução dos riscos das doenças e/ou incapacidades funcionais, ou na 
disposição para a realização das atividades diárias do indivíduo e da sociedade como um todo (3).

O nível socioeconômico é um determinante que tem sido referenciado como modulador da prática de atividade física e da aptidão física relacionada à saúde. Segundo Seabra et al. (4), embora não ainda não seja consensual, a literatura tem indicado associação positiva entre o nível socioeconômico e o engajamento em práticas de atividades físicas. Assim, o suporte social, o local de moradia, o IDH da cidade, a renda dos pais, representam desigualdades sociais na oportunidade da prática esportiva e o aprimoramento da aptidão física (5-7)

Apesar do número considerável de estudos realizados no Brasil, abordando a AFRS (8-10). Observa-se uma lacuna na literatura que a ausência de estudos que analisam a aptidão física de adolescentes que vivem em áreas com médio/baixo índice de desenvolvimento humano. Como essas áreas são típicas no Brasil, investigar a AFRS desses adolescentes poderá servir como ponto de partida para o desenvolvimento de programas de promoção da saúde. A melhoria da AFRS pode prevenir algumas das principais causas de morbi-mortalidade na população brasileira, resultando na diminuição direta dos gastos públicos com cuidados médicos emelhoria da qualidade de vida. Assim, o objetivo do presente estudo foi analisar a AFRS à saúde de adolescentes residentes em áreas com médio/baixo índice de desenvolvimento humano.

\section{MATERIAIS E MÉTODOS}

O estudo sobre a AFRS em adolescentes commédio/baixo índice de desenvolvimento humano foi desenvolvido a partir de um estudo transversal "Análise da atividade física e aptidão física relacionada à saúde em adolescentes rurais e urbanos", aprovado pelo Comitê de Ética das Faculdades Unidas do Norte de Minas (Parecer 129/09).

O presente estudo foi realizado em uma amostra representativa de adolescentes do município de Januária, MG, situado na região do Médio São Francisco. Esse município tem 65463 habitantes. O IDH é de 0,699 e classifica o município com médio-baixo desenvolvimento humano (12), a opção pelo ensino público gratuído foi para garantir a maior representativade da amostra com essa classificação.

A população do estudo foi composta por 4495 adolescentes matriculados em escolas públicas estaduais. Para o cálculo do tamanho amostral, adotou-se prevalência desconhecida para o desfecho (igual a $50 \%$ ), erro tolerável de cinco 
pontos percentuais, nível de confiança de $95 \%$, efeito de delineamento de 1,5 , acrescentando $15 \%$ para possíveis perdas e recusas. Assim, estimou-se que seria necessário coletar informações de 611 adolescentes. Em virtude das características do processo amostral por conglomerado, participaram da amostra 627 estudantes.

O processo de amostragem foi estratificado por escolas públicas do ensino fundamental e médio e conglomerado de turmas. No primeiro estágio, consideraram-se somente as escolas que tinham ensino fundamental e médio, pois eram as maiores escolas da região e concentravam a maior quantidade de alunos. Procedeu-se ao sorteio de quais escolas participariam do estudo, tendo como base uma lista fornecida pelas próprias instituições com a idade dos estudantes. No segundo estágio, foram convidados a participar do estudo todos os adolescentes de 14 a 17 anos que estavam presentes em sala de aula no dia da coleta de dados.

Todos os responsáveis pelos adolescentes, após serem informados sobre o propósito da investigação e os procedimentos a serem adotados, assinaram um termo de consentimento livre e esclarecido, autorizando a participação dos adolescentes na pesquisa.

\section{Antropometria}

A massa corporal (MC) foi obtida em uma balança digital, da marca Filizola, com resolução de $0,05 \mathrm{~kg}$, e a estatura (EST) por meio de uma trena antropométrica da marca Sanny, com resolução de $0,1 \mathrm{~cm}$, afixada na parede. Estas medidas foram mensuradas seguindo o protocolo CSEP (13). A partir dessas informações, obteve-se o índice de massa corporal $\left(\mathrm{IMC}=\mathrm{MC}_{\mathrm{kg}} / \mathrm{EST}_{\mathrm{m}}{ }^{2}\right)$.

O perímetro da cintura $(\mathrm{PC})$ foi mensurado na região de menor circunferência abdominal (14), com uma fita métrica flexível, com resolução de $0,1 \mathrm{~mm}$.

As dobras cutâneas (DC) das regiões do tríceps (TR), bíceps (BI), subescapular (SE), suprailíaca (SI) e panturrilha medial (PM) foram aferidas utilizando um plicômetro da marca Cescorf, com resolução de $0,1 \mathrm{~mm}$, seguindo os procedimentos de Avaliação do Plano Canadense de Avaliação da Atividade Física, Aptidão e Estilo de Vida (13).

Como indicador de adiposidade corporal, foram utilizadas as variáveis MC, EST, PC e DC, para determinar a zona adequada para a saúde, estimada de acordo com riscos de morbidade e mortalidade (13). 
Testes motores

Os adolescentes foram submetidos a uma bateria de testes motores que foi aplicada obedecendo a seguinte sequência: sentar-e-alcançar (flexibilidade), abdominal modificado 1 minuto (força/resistência muscular) e corrida/ caminhada de 1600 metros (aptidão cardiorrespiratória). A AFRS foi avaliada através da bateria de testes motores propostos pela AAHPERD (15) e, para a interpretação dos resultados foram utilizados os critérios sugeridos pelo Physical Best.

Análise estatística

Para caracterizar a amostra os dados dos componentes da AFRS por idade e sexo foram tratados, inicialmente, por procedimentos descritivos (média, desvio padrão). A análise de variância (ANOVA two-way) foi utilizada para as comparações entre sexo e idade. O teste post hoc de Bonferroni foi empregado para a identificação das diferenças específicas nas variáveis em que os valores de $\mathrm{F}$ encontrados foram superiores ao critério de significância estatística estabelecido $(\mathrm{p}<0,05)$. Adistribuição de frequências percentuais foi estabelecida para verificar a proporção de adolescentes que não atenderam aos critérios de saúde. Na comparação entre duas proporções, foi aplicado o teste de significância para diferenças entre as proporções. Em todas as análises adotouse nível de significância de $5 \%$.

\section{RESULTADOS}

A Tabela 1 apresenta as características antropométricas dos adolescentes (média, desvio padrão e estatística F) de acordo com o sexo e idade cronológica. Efeito isolado do sexo $(\mathrm{p}<0,05)$ foi encontrado na MC e EST, bem como efeito isolado da idade $(p<0,05)$ foi verificado em todas as variáveis analisadas. Foi observada, ainda, interação entre sexo e idade na MC e EST ( $p<0,05)$.

A Tabela 2 apresenta os valores (média, desvio padrão e estatística F) referentes aos testes de AFRS dos adolescentes, segundo o sexo e idade cronológica. Foi verificado apenas efeito isolado do $\operatorname{sexo}(\mathrm{p}<0,05)$ nas variáveis abdominal e aptidão cardiorrespiratória.

Pode ser observado na Figura 1 a proporção (\%) de adolescentes que não atenderam aos critérios de saúde estabelecidos para a composição corporal e para os três testes motores. Os resultados demonstraran que $25,1 \%$ dos adolescentes (14 a 17 anos) encontram-se com composição corporal inadequada, com proporção estatisticamente superior para o sexo masculino 
$(32,3 \%)$ em relação ao feminino (18 \%). Foi observada maior proporção de inadequação na composição corporal nas idades de 14,16, 17 anos e no conjunto das idades (14-17 anos) para o sexo masculino, quando comparado ao feminino.

Tabela 1. Características antropométricas dos escolares (média, desvio padrão e estatística F) de acordo com o sexo e idade

\begin{tabular}{ccccc}
\hline $\begin{array}{c}\text { Idade } \\
\text { (anos) }\end{array}$ & sexo & MC $(\mathrm{Kg})$ & EST $(\mathrm{cm})$ & IMC \\
\hline 14 & Masculino & $48,92 \pm 10,72^{\mathrm{a}}$ & $161,43 \pm 9,84^{\mathrm{a}}$ & $18,63 \pm 3,05^{\mathrm{a}}$ \\
& Feminino & $47,80 \pm 6,30^{\mathrm{A}}$ & $158,36 \pm 6,68^{\mathrm{A}}$ & $19,05 \pm 2,27^{\mathrm{A}}$ \\
15 & Masculino & $56,00 \pm 7,96^{\mathrm{bC}}$ & $168,30 \pm 6,76^{\mathrm{bC}}$ & $19,73 \pm 2,31^{\mathrm{ab}}$ \\
& Feminino & $51,32 \pm 7,61^{\mathrm{B}}$ & $160,68 \pm 5,70^{\mathrm{AB}}$ & $19,85 \pm 2,59^{\mathrm{AB}}$ \\
16 & Masculino & $58,77 \pm 7,86^{\mathrm{cd}}$ & $171,77 \pm 5,97^{\mathrm{cd}}$ & $19,89 \pm 2,27^{\mathrm{D}}$ \\
& Feminino & $52,56 \pm 6,92^{\mathrm{B}}$ & $161,20 \pm 6,70^{\mathrm{B}}$ & $20,24 \pm 2,53^{\mathrm{BC}}$ \\
17 & Masculino & $62,17 \pm 11,53^{\mathrm{d}}$ & $172,42 \pm 7,95^{\mathrm{d}}$ & $20,86 \pm 3,29^{\mathrm{b}}$ \\
& Feminino & $53,94 \pm 7,52^{\mathrm{B}}$ & $160,64 \pm 6,19^{\mathrm{AB}}$ & $20,88 \pm 2,52^{\mathrm{C}}$ \\
F sexo & & $55,144^{*}$ & $209,140^{*}$ & 1,135 \\
F idade & $34,369^{*}$ & $28,366^{*}$ & $14,148^{*}$ \\
F sexo x idade & $4,541^{*}$ & $10,876^{*}$ & 0,187 \\
\hline
\end{tabular}

MC:Massa Corporal; EST: Estatura; IMC: Índice de Massa Corporal; a,b,c- comparação entre as idades no sexo masculino; $A, B, C$-comparação entre as idades no sexo feminino; $A A, B B, C C$ - não há diferença significativa entre as idades $(p>0,05) ; A B, B C, a b, b c-$ diferença significativa entre as idades $(p<0,05)$.

Tabela 2. Desempenho dos escolares (média, desvio padrão e estatística F) em testes motores de acordo com o sexo e idade

\begin{tabular}{ccccc}
\hline $\begin{array}{c}\text { Idade } \\
\text { (anos) }\end{array}$ & Sexo & FLEX $(\mathrm{cm})$ & ABD (rep) & T1600 (m) \\
\hline 14 & Masculino & $26,27 \pm 6,00$ & $25,78 \pm 6,79$ & $8,31 \pm 2,34$ \\
& Feminino & $25,31 \pm 7,99$ & $16,75 \pm 6,91$ & $11,57 \pm 2,52$ \\
15 & Masculino & $27,07 \pm 7,77$ & $26,47 \pm 7,26$ & $9,00 \pm 2,56$ \\
& Feminino & $25,94 \pm 8,02$ & $18,66 \pm 7,75$ & $12,12 \pm 3,59$ \\
16 & Masculino & $25,87 \pm 8,36$ & $25,69 \pm 8,08$ & $8,59 \pm 2,13$ \\
& Feminino & $26,08 \pm 8,12$ & $17,39 \pm 7,84$ & $11,59 \pm 3,07$ \\
17 & Masculino & $25,73 \pm 8,08$ & $25,92 \pm 8,73$ & $8,99 \pm 2,57$ \\
& Feminino & $25,09 \pm 7,82$ & $17,85 \pm 7,42$ & $11,94 \pm 2,97$ \\
F sexo & & 0,959 & $176,733^{*}$ & $172,936^{*}$ \\
F idade & & 0,519 & 0,866 & 1,638 \\
F sexo x idade & 0,229 & 0,174 & 0,079 \\
\hline
\end{tabular}

FLEX: flexibilidade; ABD: força/resistência abdominal; T1600: aptidão cardiorrespiratória. cm: centímetros; rep: repetições; $m$ : metros; * $\mathrm{p}<0,05$ na comparação entre os sexos.

Na flexibilidade, observou-se que 40,8 \% dos adolescentes não atingiram os critérios estabelecidos para a saúde. Diferença significante foi encontrada entre os sexos na idade de 14 anos, sendo a proporção de inadequação maior no feminino que no masculino $(\mathrm{p}<0,05)$.

Para a força/resistência muscular, observou-se que 98,5\% dos adolescentes encontraram-se classificados abaixo dos critérios de saúde. Diferença 
significante foi encontrada na comparação entre as proporções na idade de 15 anos $(p<0,05)$. Esses resultados indicaram maior proporção de adolescentes do sexo feminino $(99,2 \%)$ que do masculino $(97,7 \%)$ abaixo dos critérios mínimos recomendados para a saúde.

Figura 1. Proporção (\%) de escolares que não atenderam aos critérios de saúde estabelecidos à composição corporal e para os testes motores de acordo com sexo e idade. * $p<0,05$ para comparação entre os sexos
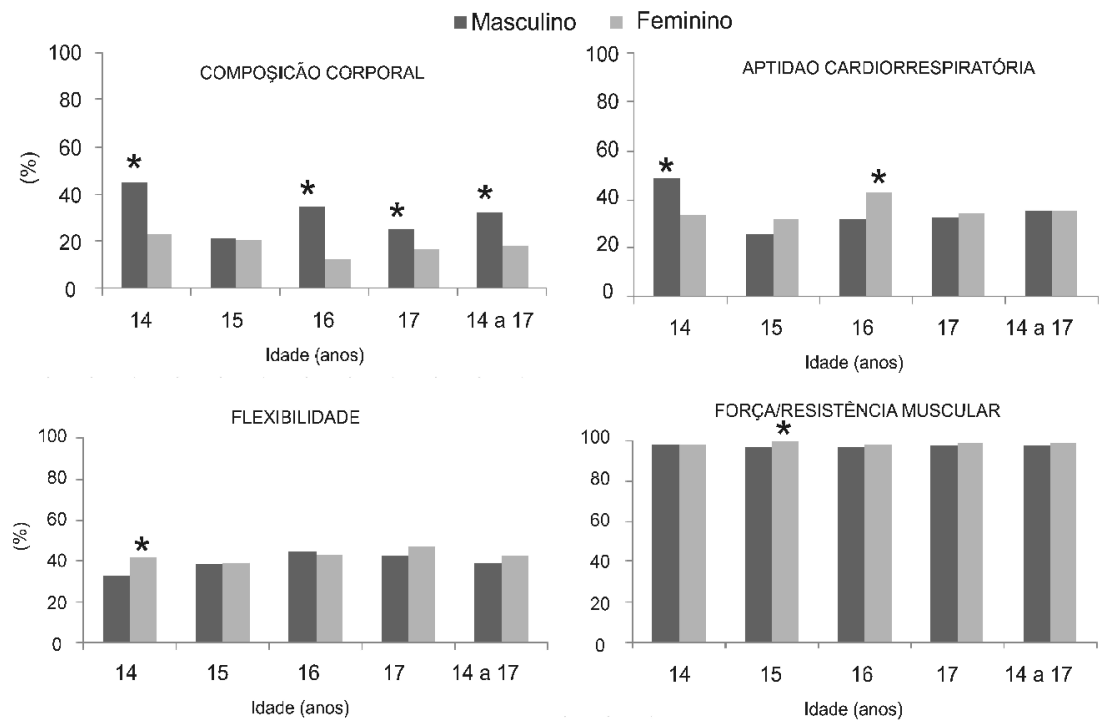

Na aptidão cardiorrespiratória, 35,4\% dos adolescentes tiveram desempenho abaixo do estabelecido para a saúde. Diferenças entre os sexos nas proporções foram verificadas nas idades de 14 e 16 anos. Esses achados apontam uma maior proporção de adolescentes do sexo masculino na idade de 14 anos, e do feminino na idade de 16 anos que não atenderam aos critérios mínimos recomendados para a saúde.

Em relação à classificação geral, observou-se elevada prevalência de adolescentes (masculino 99,6 \% e feminino $100 \%$ ) que não atenderam, simultaneamente, aos critérios de saúde estabelecidos pela proposta adotada nos três testes motores.

\section{DISCUSSÃO}

O principal achado deste estudo foi identificar uma elevada prevalência deAFRS, abaixo dos critérios recomendados à saúde em adolescentes com médio/baixo 
índice de desenvolvimento humano, independentemente do sexo e da idade. Esta constatação adiciona à literatura informações podem ser úteis no planejamento, implantação e orientações de intervenções específicas para esta população.

A análise da gordura corporal em componente da AFRS, sugere que um em cada quatro adolescente tem a composição corporal inadequada. Pesquisa realizada em adolescentes de Londrina-PR (15 a 18 anos) revelou que $16 \%$ das moças e $26 \%$ dos rapazes apresentam composição corporal inadequada para a saúde (16). Os resultados do presente estudo são similares aos relatos de outros pesquisadores (17), quando analisaram a adequação aos critérios recomendados à saúde pelo Physical Best em escolares de 10 e 11 anos de idade de baixo nível socioeconômico.

Estudos recentes observaram que a obesidade está significativamente associada com sexo, idade e região. Sendo mais expostos à obesidade os adolescentes do sexo masculino, nas idades de 10 a 13 anos, residentes nas regiões Sul e Sudeste (18).

Quanto a flexibilidade, verificou-se que $40,8 \%$ dos adolescentes não atingiram os critérios recomendados para a saúde (feminino $=42,4 \%$; masculino=39,1\%). Esses resultados corroboram estudos prévios em escolares de baixo nível socioeconômico (18). Não obstante, essa prevalência foi superior à encontrada na cidade de LondrinaPR, onde 20,5\% dos adolescentes (17) e $33 \%$ das crianças não atingiram os critérios mínimos recomendados para a saúde, com adolescentes de alto nível socioeconômico (19). O número expressivo de adolescentes que não atendeu aos critérios estabelecidos para a saúde constitui-se em preocupação, pois flexibilidade e força abdominal reduzidas estão associadas às lombalgias (20), consideradas uma das maiores causas da incapacidade funcional.

No componente de aptidão física relacionada à saúde relacionada à força/ resistência muscular (abdominal), verificou-se elevada proporção de adolescentes que não atenderam aos critérios estabelecidos à saúde $(98,4 \%)$. Esses resultados foram superiores aos encontrados em pesquisas com adolescentes brasileiros residentes em cidades com IDH mais elevado $(16,20)$.

Quanto ao componente da aptidão cardiorrespiratória, os achados do presente estudo demonstraram que apenas um de cada três adoloescentes atenderam aos critérios recomendados para a saúde. Em outra pesquisa realizada em JequiéBA, (IDH 0,609) observou-se que 85,0 \% e 79,0 \% dos meninos e meninas, 
respectivamente, ficaram abaixo dos pontos de corte preconizados à saúde (10). Outro estudo (21), realizado com escolares residentes em Chapecó, SC (IDH 0,76 ), sugeriu que $43,7 \%$ dos adolescentes estavam exportos ao desenvolvimento de doença crônico-degenerativa associada a baixos níveis de aptidão cardiorrespiratória. Outra investigação relatou nem mesmo os adolescentes de hábitos de lazer ativo obtiveram classificação dentro da zona saudável para a aptidão cardiorespiratória (11).

No presente estudo, considerando juntos os três componentes da AFRS, notouse que $99,8 \%$ dos adolescentes não atenderam aos critérios mínimos recomendados para a saúde. Recentemente, Glaner (22) relatou que $87,1 \%$ e 93,3 \% dos adolescentes da área rural e urbana, respectivamente, não atendem aos critérios recomendados para a saúde.

As principais limitações do presente estudo estão relacionadas ao delineamento de corte transversal, que não permite identificar as relações de causalidade; o uso de critérios de classificação baseado em pontos de corte estabelecidos em outros países; não inclusão de estudantes de escolas privadas. Todavia os resultados observados no presente estudo possuem validade externa para adolescentes com características semelhantes, as quais destacam-se: adolescentes de ensino público e gratuito mantido pelo Estado; residentes em regiões com médio/baixo IDH.

De acordo com as informações produzidas nesse estudo, conclui-se que a AFRS dos adolescentes residentes em áreas com médio/baixo IDH é inadequada, evidenciando riscos à saúde, independente do sexo e idade. Neste sentido, acreditase que seja de fundamental importância a implementação de intervenções sociais direcionadas à adolescencia que possam interferir diretamente nos níveis de AFRS. Além disso, esses achados podem ser importantes para embasar as diretrizes de políticas públicas de promoção de saúde no ambiente escolar.

Enfatiza-se a importância de realização de outras pesquisas na região de Januária-MG, considerando aspectos como o estilo de vida, hábitos alimentares e prática regular de atividade física, para melhor compreender as questões que interferem na realidade encontrada. Vale ressaltar que essa pesquisa foi a primeira a ser desenvolvida com adolescentes de escolas públicas em Minas Gerais, com enfoque na AFRS

Agradecimentos: Capes Foundation-Proc. nº. BEX 0951/10-2. 


\section{REFERÊNCIAS}

1. Bouchard C, Després JP. Physical Activity and Health: Atherosclerotic, Metabolic, and Hypertensive Diseases. Res Q Exerc Sport 1995; 66(4):268-75.

2. Pate RR. The evolving definition of physical fitness. Quest 1988; 40(3):174-9.

3. American college of sports medicine (ACSM). Manual do ACSM para avaliação da aptidão física relacionada à saúde. Rio de Janeiro: Guanabara Koogan; 2006.

4. Seabra AF, Mendonça DM, Thomis MA, Anjos LA, Maia FA. Determinantes biológicos e sócio-culturais associados à prática de atividade física de adolescentes. Cad Saúde Pública. 2008; 24:721736.

5. Kimhi A. Socio-Economic Determinants of Health and Physical Fitness in Southem Ethiopia. Econ Hum Biol. 2003; 1:55-75.

6. La Torre G, Masala D, De Vito E, Langiano E, Capelli G Ricciardi W. Extra-curricular physical activity and socioeconomic status in Italian adolescents. BMC Public Health. 2006, 6:22 doi:10.1186/14712458-6-22.

7. Cerin $E$, Leslie $E$. How socio-economic status contributes to participation in leisuretime physical activity. Social Sci Med. 2008; 66:2596-2609.

8. Farias ES, Carvalho WRG, Gonçalves EM, Guerra-Júnior G Efeito da atividade física programada sobre a aptidão física em adolescentes adolescentes. Rev Bras Cineantropom Desempenho Hum. 2010; 12(2):98-105.

9. Nascimento TB, Pereira DC, Glaner MF. Prevalência de indicadores de aptidão física associada à saúde em adolescentes. Motriz. 2010; 16(2):387-94.

10. Dorea V, Ronque ERV, Cyrino ES, Serassuelo Júnior H, Gobbo LA, Carvalho FO et al. Aptidão física relacionada à saúde em adolescentes de Jequié, BA, Brasil. Rev Bras Med Esporte. 2007; 14(6):494-9.

11. Sales-Nobre FS, JomadA-Krebs R. Valentini NC. Práticas de lazer, nível de atividade física e aptidão física de moças e rapazes brasileiros. Rev Salud Pública (Bogotá) 2009; 11(5):713-23.

12. Programa das Nações Unidas para o Desenvolvimento (PNUD) [Internet]. Ranking do Índice de Desenvolvimento Municipal dos municípios do Brasil, 2000. Available at: http://www.pnud.org.br/ atlas/tabelas/index.php. Accessed 15 Septiembre, 2009.

13. Canadian Society for Exercise Physiology (CSEP). The Canadian Physical Activity, Fitness and Lifestyle Appraisal: CSEP's guide to health active living. 2. ed. Ottawa: CSEF; 1998.

14. Petroski EL. Antropometria: Técnicas e padronizações. $3^{a}$ ed. Blumenal, Nova Letra; 2007. p. 57-69.

15. American Alliance for Health, Physical Education, Recreation and Dance (AAHPERD). Physical best. Reston: American Alliance for Health, Physical Education, Recreation and Dance; 1988.

16. Guedes DP, Guedes JERPG, Barbosa DS, Oliveira JA. Atividade física habitual e aptidão física relacionada à saúde em adolescentes. Rev Bras Ciênc Mov. 2002; 10(1):13-21.

17. Serassuelo Junior H, Rodrigues AR, Cyrino ES, Ronque ERV, Oliveira SR Simões AC. Aptidão física relacionada à saúde em adolescentes de baixo nível socioeconômico do município de Cambél PR. Rev Educ Fís. 2005; 16(1):5-11.

18. Pelegrini A, Petroski EL, Coqueiro RS, Gaya ACA. Overweight and obesity in brazilian schoolchildren aged 10 to 15 years: data from a Brazilian sports project. Arch Lat-am Nutric. 2008; 58(4):34398.

19. Ronque VER, Cyrino ES, Dorea VR, Serassuelo Junior H, Galdi EHG, Arruda M. Diagnóstico da aptidao fisica em adolescentes de alto nivel socioeconomico: avaliação referenciada por criterios de saude. Rev Bras Med Esporte. 2007; 13(2):71-5.

20. Nadler SF, Malanga GA, Feinberg JK, Prybicienm M, Stitik TP, Deprince M. Relationship between hip muscle imbalance and occurrence of low back pain in collegiate athletes: a prospective study. Am J Phy Med Rehabil. 2001; 80(8):572-7.

21. Glaner MF. Aptidão física relacionada à saúde de adolescentes rurais e urbanos em relação a critérios de referência. Rev Bras Educ Fís Esporte. 2005; 19(1):13-24.

22. Glaner MF. Nível de atividade física e aptidão física relacionada à saúde em rapazes rurais e urbanos. Rev Paul Educ Fís. 2002; 16(1),76-85. 